\title{
Combining reverse osmosis and pulsed electrical current electrodialysis for improved recovery of dissolved organic matter from seawater
}

\author{
B. K. Gurtler ${ }^{\mathrm{a}}$, T. A. Vetter ${ }^{\mathrm{a}, 1}$, E. M. Perdue ${ }^{\mathrm{b}}$, E. Ingall ${ }^{\mathrm{b}}$, J.-F. Koprivnjak $^{\mathrm{b}, 2}$, P. H. Pfromm ${ }^{\mathrm{a}, \mathrm{c}}$ \\ ${ }^{a}$ Department of Chemical Engineering, Kansas State University, 1005 Durland Hall, Manhattan, \\ Kansas, 66506-5102, USA ${ }^{\mathrm{b}}$ School of Earth and Atmospheric Sciences, Georgia Institute of \\ Technology, 311 Ferst Drive, Atlanta, Georgia, 30332-0340, USA
}

Published in Journal of Membrane Science, 332(2), 328-336, 2008

\begin{abstract}
Dissolved organic carbon (DOC) in the oceans is one of the largest dynamic reservoirs of carbon on earth, comparable in size to the atmospheric reservoir of carbon $\left(\right.$ as $\left.\mathrm{CO}_{2}\right)$ in the atmosphere, or to the amount of carbon in all terrestrial and aquatic biota. The concerted efforts of earth scientists, atmospheric scientists, and biologists who study global biogeochemical cycles and the earth's climate have yielded a rather detailed understanding of carbon in the atmosphere and in biota. Marine dissolved organic matter (DOM) is far less well characterized, principally because it exists as a highly diluted mixture of perhaps millions of organic compounds in a highly saline aqueous solution. Prior to 2007, only around $1 / 3$ of marine DOM was typically recovered from seawater for research purposes, regardless of the method of isolation. In 2007, reverse osmosis (RO) and electrodialysis (ED) were coupled to achieve recoveries of $64 \%-93 \%$ of marine DOM. The level of residual salts in the concentrated samples, however, still precluded the characterization of marine DOM by solid-state NMR, mass spectrometry, or even elemental analysis. This paper describes a major improvement to the RO/ED method, in which pulsed electrodialysis is used (at sea) to reach roughly 100-fold greater removal of salts compared to not pulsed ED while maintaining comparable recoveries of DOM.
\end{abstract}

Keywords: electrodialysis, reverse osmosis, dissolved organic carbon, ocean, pulsed

${ }^{c}$ : to whom correspondence shall be addressed, e-mail pfromm@ksu.edu, phone 785-532-4312, fax 785-532-7372

${ }^{1}$ : now with Koch Industries, Wichita, Kansas, USA

2: now at Trent University, Ontario, Canada 


\section{Introduction}

The overall goal of our work is to develop a practical membrane-based process that can recover dissolved organic matter (DOM) from seawater aboard an oceangoing research vessel for subsequent scientific investigation. The DOM must be reasonably free of salts and multimilligram samples must be recovered in concentrated form from a given sampling location and depth in the ocean. The samples must be small in volume to be frozen for later analysis. In this paper we describe a significant improvement on our initial process ${ }^{1}$ : the application of pulsed electrodialysis to improve the terminal desalination of the samples while maintaining high DOM recoveries.

\section{Marine DOM in the global carbon cycle}

Dissolved organic matter in the oceans is one of the most significant reservoirs of organic carbon (C) on Earth. Marine DOM contains approximately 50\% (w/w) carbon, and it is commonly quantified as dissolved organic carbon (DOC). Here DOC is used when referring to quantities or concentrations of $\mathrm{DOM}^{1}$. Marine DOC reservoirs contain approximately 700 gigatons ( $\mathrm{Gt}, 1 \mathrm{Gt}=10^{9}$ metric tons) of $\mathrm{C}$, which is comparable to the $\mathrm{C}$ present in atmospheric $\mathrm{CO}_{2}(750 \mathrm{Gt})$ and in all terrestrial and aquatic biota $(573 \mathrm{Gt})^{2}$. This illustrates the importance of marine DOM for scientists interested in global geochemical cycles and climate change.

\section{Rationale for obtaining isolated samples of marine DOM}

Marine DOM may be viewed conceptually as a transitional material that lies somewhere along the diagenetic vector that leads from marine biomass to carbon dioxide. It is anticipated that spatial and temporal variations in the average oxidation state of $\mathrm{C}$ in marine $\mathrm{DOM}$ will reflect the extent to which a given sample of DOM has been modified through photodegradation 
and biodegradation as it transported through space and time in the world's oceans. The average oxidation state of $\mathrm{C}$ in marine DOM is determined primarily by the stoichiometric proportions of organically bound $\mathrm{H}$ and $\mathrm{O}$, because those elements are much more abundant than $\mathrm{N}, \mathrm{P}$, and $\mathrm{S}$ in marine DOM. Organically bound $\mathrm{H}$ and $\mathrm{O}$ cannot be measured on aqueous solutions of DOM, and accurate elemental analyses that include $\mathrm{H}$ and $\mathrm{O}$ are only possible if freeze-dried DOM samples contain less than around 5wt\% ash (e.g., residual sea salts). For this reason, less than 20 such elemental analyses of marine DOM could be found in a recent review of the literature ${ }^{3}$, and all of those analyses were conducted on the relatively minor fraction of marine DOM that can be isolated using adsorption on XAD resins (a family of relatively hydrophobic adsorbents manufactured by Rohm and Haas - see later discussion).

The recovery of DOM from the ocean in sufficient quantity (hundreds of mg per sample location and depth), purity, and concentration to enable basic elemental analyses and much more sophisticated analyses is a very significant challenge, especially when considering that contamination or alteration of the DOM during processing must be avoided. Because DOM may be altered by both photodegradation and biodegradation, shipboard processing of seawater is the preferred means of rapidly recovering and conserving the samples. Storage and/or transport of large amounts of seawater for off-site processing is impractical and raises questions regarding unintended changes in the DOM. Seawater typically contains around 35,000 $\mathrm{mg} \mathrm{l}^{-1}$ of dissolved sea salts and $0.5-1.0 \mathrm{mg}^{-1}$ of DOC, the lower DOC concentrations being found in the deeper ocean (most of the ocean reservoir). The mass ratio of sea salts to DOM in seawater (assuming that DOM DOC x 2) is 17,500:1 or higher. To obtain representative samples of isolated marine DOM that are suitable for the widest array of methods of chemical and spectroscopic characterization, three obstacles must be overcome: virtually complete removal of the salts in 
seawater, removal of the bulk of the water, and retention of DOM, which consists of a spectrum of molecules with molecular weights from several 10's to several $1000 \mathrm{gram} / \mathrm{mol}$.

\section{Earlier methods for isolation of marine DOM}

Prior to 2007, nearly all efforts to isolate suitable samples of marine DOM used XAD resins, $\mathrm{C}_{18}$ adsorbents, or cross-flow ultrafiltration. In the vast majority of such studies, less than $1 / 3$ of marine DOM was recovered ${ }^{3,4}$. All of these methods are known to yield biased samples. Samples that are isolated using either XAD resins or $\mathrm{C}_{18}$ adsorbents are disproportionately enriched in both chromophores and fluorophores, and samples isolated by ultrafiltration are necessarily enriched in higher molecular weight components of marine DOM. Furthermore, samples that are isolated using $\mathrm{XAD}$ resins or $\mathrm{C}_{18}$ adsorbents are commonly adsorbed from seawater at $\mathrm{pH} 2-2.5$, and they are subsequently eluted from the stationary phase with either highly alkaline solutions of $\mathrm{NaOH}$ and/or an organic solvent such as methanol. The exposure of marine DOM to such chemical reagents and conditions raises concern that isolated samples of marine DOM might have been modified chemically by the isolation method. This problem is avoided when ultrafiltration is used to isolate marine DOM; however, those samples still contain a large proportion of residual sea salts, even after exhaustive diafiltration. It is common to obtain isolated samples by ultrafiltration for which the ratio of residual sea salts to DOM is at least 5:1. The elemental compositions of such samples (e.g., C, H, O, N, S, and P) cannot be measured accurately, although $\mathrm{C} / \mathrm{N}, \mathrm{C} / \mathrm{P}$, and N/P molar ratios can be measured. Overall, it can be concluded that the isolation of marine DOM using $\mathrm{XAD}$ resins, $\mathrm{C}_{18}$ adsorbents, and ultrafiltration membranes has not resulted in high yields of representative samples of isolated marine DOM that are suitable for the widest array of methods of chemical and spectroscopic characterization. 
Reverse osmosis for removal of water

Reverse osmosis ${ }^{5}$ (RO) is used to desalinate seawater for human consumption on the industrial scale. RO has also been shown to be an effective method for isolation and concentration of DOM from fresh waters. A significant fraction (on the order of $90 \%$ or more) of DOC from river water can be recovered and concentrated by $\mathrm{RO}$ with little physical or chemical alteration prior to in-depth analysis ${ }^{6,7}$. Unfortunately, RO membranes also retain and concentrate the salts and minerals in natural waters. When processing relatively dilute fresh waters, this problem is largely eliminated when RO is used in conjunction with water softening by $\mathrm{H}^{+}$-saturated cation exchange resins. Seawater contains around $35 \mathrm{~g} \mathrm{~L}^{-1}$ of dissolved salts, so its osmotic pressure is much greater than that of a typical fresh water. It is possible to use RO to extract relatively pure water from seawater if an RO system is operated at sufficiently high pressure to overcome the osmotic pressure of seawater. The selective removal of water increases the concentrations of all aqueous solutes and leads quickly to supersaturation with respect to one or more sparingly soluble salts (e.g. $\mathrm{CaSO}_{4} \cdot 2 \mathrm{H}_{2} \mathrm{O}(\mathrm{s})$ and $\mathrm{CaCO}_{3}(\mathrm{~s})$ ). Precipitation of supersaturated solids leads to rapid fouling of RO membranes, which greatly decreases the rate of production of pure water. For all of these reasons, the direct application of RO for isolation and concentration of DOM from seawater is not practical.

Coupled reverse osmosis and electrodialysis for removal of water and sea salts

Electrodialysis $^{8}$ (ED) is a well-established method for removal of electrolytes from aqueous solutions. $\mathrm{RO}$ and $\mathrm{ED}$ have been combined to remove inorganic ions and concentrate $\mathrm{DOM}_{\text {from freshwaters }}{ }^{9}$ and a refined $\mathrm{RO} / \mathrm{ED}$ process resulted in extremely pure DOM samples at high recovery ${ }^{10}$. In 2007, the coupled RO/ED method was used to isolate DOM from seawater ${ }^{1}$. This method has enabled the relatively rapid, gentle removal of around $97 \%$ of water 
and $99.3 \%$ of sea salts (as conductivity $\mathrm{X}$ volume), while recovering an average of $76 \% \pm 15 \%$ of DOC (as concentration X volume). In Table 1 of the 2007 paper, the conductivity of the third sample was tabulated incorrectly as 10.0 millisiemens per $\mathrm{cm}\left(\mathrm{mS} \mathrm{cm}^{-1}\right)$. The correct value is $9.0 \mathrm{mS} \mathrm{cm}{ }^{-1}$. Volumetric re-calibration of the entire RO/ED system indicates that sample volumes were approximately $198 \mathrm{~L}$. Even though the recovery of DOC by the RO/ED method easily doubles or triples most results that have been obtained using XAD resins, $\mathrm{C}_{18}$ adsorbents, or ultrafiltration, the average conductivity of the final isolated solutions $\left(9.5 \mathrm{mS} \mathrm{cm}{ }^{-1}\right)$ is at least 60 times greater than the conductivity of the world-average river (around $150 \mu \mathrm{S} \mathrm{cm}^{-1}$ ). Assuming that the mass concentrations of sea salts $\left(\mathrm{mg} \mathrm{l}^{-1}\right)$ and the conductivities of samples $\left(\mathrm{mS} \mathrm{cm}^{-1}\right)$ are reduced by the same percentage during the RO/ED process and that as a rule of thumb DOM DOC $\mathrm{x} 2$, then the mass ratio of sea salts to DOM in the final isolated samples is around 180:1. Such samples are certainly not optimal for either elemental analysis or advanced chemical and spectroscopic characterization.

Further removal of sea salts with little or no additional loss of DOC is a necessary next step in the development of the RO/ED method, and this paper describes the development and implementation (at sea) of that step through the use of pulsed (on/off) electrodialysis in the final phase of the RO/ED process. Some pertinent work on pulsed ED is discussed below.

\section{Potential advantages of pulsed electrodialysis}

Fouling of ion exchange membranes is a serious issue but most prior work deals with (relative to seawater) very high concentrations of potential foulants ${ }^{11}$. A recent report on the application of pulsed on/off ED with pulse lengths of several seconds to demineralize casein solutions concludes that protein fouling of the membranes was significantly diminished compared to ED without pulsing ${ }^{12}$. The authors speculate that the "relaxation time" (electrical 
ED current off) prevents fouling. This supports the results that follow and the hypothesis that the anionic DOM components are less prone to foul or penetrate the anion exchange membrane during pulsed ED, when compared to continuous ED.

An experimental study of the separating capacity of a membrane to ions of like but unequal charge $\left(\mathrm{Na}^{+}\right.$and $\left.\mathrm{Ca}^{2+}\right)$ by $\mathrm{ED}$ under pulsed electrical current has been reported ${ }^{13}$. The DC pulses used in these experiments ranged from $10^{-3}$ to 5 seconds. When using the asymmetrically pulsed (on/off) mode, the transport of ions through the membrane decreased as the "on" pulse length was increased to 5 seconds while the "off" pulse length was kept constant at 1 second. This may be due to the depletion of the initially ion-rich boundary layer near the membrane when the "on" pulse is prolonged. It was concluded in this work that, with the use of a DC on/off pulsed electrical current, the transport of specific ions through the membrane can be controlled by adjusting the pulse frequency. Similarities between this research and the current study can be seen in the type of pulsed electrical current used, and the length of time that the electrical current is pulsed. Another similarity is the effort to discriminate between ions of different charge and size. Our target is to remove $\mathrm{SO}_{4}{ }^{2-}$ and $\mathrm{Cl}^{-}$and retain organic DOM anions.

The effects of pulsed electric fields (square wave of on/off pattern with 60, 100, and $200 \mathrm{~Hz}$ ) on membrane fouling in $\mathrm{ED}$ were studied with a solution of $\mathrm{NaCl}$ containing humate ${ }^{14}$. Humate is an unspecified (but probably $\mathrm{Na}^{+}$) salt of humic acid - a highly complex mixture of organic acids that can be extracted from soils and sediments. Humic acid and humate are perhaps somewhat comparable to the marine DOM in this research, both in their natural origin and their compositional complexity. It was found that the pulsed electric fields enhanced the mobility of all particles in the fouling layer at the membrane surface and decreased the electrical resistance of the ED cell. Fouling effects were minimized in the pulsed mode at $100 \mathrm{~Hz}$, 
implying that the mobilities of organic ions in the boundary layer near the membrane are increased by application of a square wave electrical current. By reducing the concentration of organic anions in the boundary layer, the selectivity of the ED process for smaller, more rapidly diffusing inorganic ions is increased. Similarities can be seen between this research and the work presented here because the inorganic solute $\mathrm{NaCl}$ is targeted to be removed in the presence of humate (analogous to desalting seawater in presence of DOM). A major difference, however, was the much higher pulse frequency of $60-200 \mathrm{~Hz}$, in contrast to a pulse frequency of about $0.25 \mathrm{~Hz}$ in this research. A symmetrical on/off sequence with pulses on the order of seconds is suggested elsewhere ${ }^{15}$ and is supported by our qualitative observation of equilibration times after changing stack voltages.

\section{Using pulsed ED in the coupled RO/ED method for isolation of DOM from seawater}

A comparison of experiments for the recovery of concentrated DOM from ocean water using the RO/ED method with and without pulsed electrical current is reported here. The pulsed ED method is here explored at final diluate electrical conductivities far below the usual range of electrodialysis. All experiments were performed on shipboard during two research cruises in the Atlantic Ocean. The evidence indicates that the potential of the coupled RO/ED method for isolation and concentration of marine DOM in very low conductivity samples is significantly enhanced if electrical current is pulsed in the final ED phase of the process (see the next section for a more complete description of the RO/ED process). With this modification, it is possible to much more completely desalt seawater samples, while maintaining the previously reported excellent recovery of DOC. This low salt content enables one-step, post-processing of the concentrated DOM by freeze drying and also facilitates the subsequent sophisticated analyses of marine DOM that are routinely applied to DOM from less saline source material. 


\section{Experimental}

\section{Collection of seawater samples}

The shipboard tests were performed aboard the Research Vessel Savannah during a July, $2006^{1}$ and October, 2006 cruise in the open Atlantic Ocean off the coast near Savannah, Georgia (sampling locations are given in Table 1). Seawater samples were retrieved using generally recognized oceanographic techniques (Niskin bottles, sampling locations determined by global positioning system). The fresh seawater samples were transferred directly from Niskin bottles to 24 L HDPE carboys and then to 230 L HDPE holding tanks that were shielded from sunlight by opaque, reflective covers. Prior to processing in the RO/ED system, particulate matter was removed from seawater samples by filtration through $0.45 \mu \mathrm{m}$ polypropylene filters (Flotrex, Osmonics, Inc., Minnetonka, MN). The first $20 \mathrm{~L}$ of each filtered sample were discarded, having been used to wash the filter. More details on sample retrieval and initial treatment can be found elsewhere ${ }^{1}$.

\section{The RO/ED process for isolation of DOM from seawater}

The RO/ED process for isolating low-salt, concentrated samples of DOM from seawater is outlined in Figure 1. Before every run on shipboard, the RO/ED system, tanks, and lines were first rinsed in a once-through mode with approximately $50 \mathrm{~L}$ of the same filtered seawater that was to be processed. After the rinse, a nominally $200 \mathrm{~L}$ sample was transferred to the RO/ED sample processing tank. At the beginning of each run, the ED concentrate circulation tank was filled with an approximately 1:10 mixture of concentrate from the previous experiment and RO permeate. An electrode rinse solution for ED was prepared by dissolving $500 \mathrm{~g}$ of $\mathrm{NaH}_{2} \mathrm{PO}_{4} \cdot \mathrm{H}_{2} \mathrm{O}$ in $16 \mathrm{~L}$ of RO permeate. This solution was used for all shipboard trials. 
The coupled RO/ED process consists of three distinct phases, beginning with an initial ED phase to reduce the conductivity of the seawater sample from above $50 \mathrm{mS} \mathrm{cm} \mathrm{cm}^{-1}$ to about $15 \mathrm{mS} \mathrm{cm}^{-1}$ or less. At the end of the initial ED phase, the osmotic pressure of the sample is low enough for removal of water by $\mathrm{RO}$, so an $\mathrm{RO} / \mathrm{ED}$ phase is then used to remove water until the target volume of the sample $(<10 \mathrm{~L})$ is reached, while using ED to simultaneously remove salts to hold the conductivity roughly constant. At the end of the RO/ED phase, the RO system is drained completely, and all recovered sample is returned to the sample processing tank for further desalting in the final ED phase. In the final ED phase, the conductivity of the nowconcentrated DOM sample ( 40 ppm of DOM) is reduced to its final target value $\left(10 \mathrm{mS} \mathrm{cm}^{-1}\right.$ in the July, 2006 cruise and $0.1 \mathrm{mS} \mathrm{cm}^{-1}$ or less in the October, 2006 cruise). At the end of the final ED phase, the sample is drained completely from the ED system and immediately frozen.

After recovering the concentrated sample a $3 \mathrm{~L}$ solution of $0.01 \underline{\mathrm{M}} \mathrm{NaOH}$ is circulated for several minutes through the RO system to recover adsorbed DOM, drained completely, and then used to rinse the ED system for several minutes. After draining the rinse solution as completely as possible from the ED system, the rinse solution is immediately frozen. The yields of DOC that are discussed later in this paper include the DOC that remained in solution throughout the $\mathrm{RO} / \mathrm{ED}$ process and the DOC that is recovered by the rinse solution. The experimental details below refer to the October 2006 cruise, where pulsed electrical current ED was used for the final ED phase. More experimental details of the July 2006 cruise are given elsewhere ${ }^{1}$.

\section{Overall system design}

The basic process design of the coupled RO/ED system is shown in Figure 2. Up to $200 \mathrm{~L}$ of seawater can be processed in the sample processing tank (210 L HDPE tank with conical bottom), which is connected via shut-off valves to pumps that supply the sample to both 
the RO and ED systems. The shut-off valves control the flow of sample to the RO and ED systems. During the initial ED phase, sample was circulated through only the ED system (July, 2006) or through both the RO and ED systems (October, 2006). During the RO/ED phase, sample was circulated through both the RO and ED systems. During the final ED phase, sample was circulated through only the ED system.

During the RO/ED phase, the RO system was pressurized to remove water, which is transferred to the permeate collection tank. Permeate solutions were collected and re-used for dilution of concentrate solutions, preparation of reagents, etc. During all three phases of operation, the sample was pumped through the ED system, where salts were transferred to the ED concentrate (230 L HDPE tank). Whenever the salinity of the concentrate approached the salinity of the sample, the solution in the concentrate circulation tank was diluted approximately ten-fold with RO permeate water to maintain a favorable concentration gradient for salts between the sample (diluate) and concentrate solutions. The cathode and anode of the ED system were rinsed with an electrode rinse solution (24 L HDPE tank) that was circulated continuously between the electrode rinse tank (not shown in Figure 2) and the ED system during all three phases of the RO/ED process.

A noteworthy improvement in the sample processing tank was made after the July, 2006 cruise. The sample processing tank is a $210 \mathrm{~L}$ cylindrical, high-density polyethylene (HDPE) tank with a conical bottom. Sample is pumped from the tank through a bulkhead fitting at the bottom of the tank. A $1 / 2$ inch bulkhead fitting was installed in the sample processing tank during the July, 2006 cruise. As the sample was pumped to the RO and/or ED systems, strong vortices developed in this tank, causing air to be pulled into the pumps. This is detrimental because the dead volume of the system dictates the maximum DOC enrichment at a given initial sample 
volume. This problem was eliminated in the October, 2006 cruise by installing a 6 inch diameter circular baffle to block vertical movement of water into the bulkhead fitting and eliminate vortices. Samples could be concentrated to a smaller final volume during the October, 2006 cruise than was possible during the July, 2006 cruise.

\section{Reverse osmosis system}

A commercial RO element (Dow FilmTec TW30-4021, The Dow Chemical Company, Midland, MI) that is designed for processing tap water was used in this study. The spiral-wound element is a thin-film composite membrane consisting of three layers: a polyester support web, a microporous polysulfone interlayer, and an ultra-thin aromatic polyamide barrier layer on the top surface. The RO element has a diameter of 4 inches and a length of 21 inches, and it is housed in a fiberglass pressure vessel. A stainless steel rotary vane pump powered by a $1 \mathrm{HP}$ electric motor (type 82395K88, McMaster-Carr, Cleveland, OH) was used to supply $1200 \mathrm{~L} \mathrm{hr}^{-1}$ of sample to the RO element at a pressure of $1380-1725 \mathrm{kPa}(200-250 \mathrm{PSI})$. Stainless steel tubing and fittings were used to construct the RO system, and it was connected to the sample processing tank using reinforced ethyl vinyl acetate (EVA) tubing (type 5632K34, McMasterCarr, Cleveland, $\mathrm{OH})$.

A second RO system essentially identical to the system used to process seawater provided a quite convenient means of producing the necessary significant amounts of relatively pure water (RO permeate) from the ship's supply of drinking water. This RO permeate was used as reagent water, rinse water, and especially to lower the conductivity of the ED concentrate solution as needed during the ED process. 


\section{Electrodialysis system}

Neosepta AMX (strongly basic, 2.0-3.5 $\mathrm{Ocm}^{2} @ 25^{\circ} \mathrm{C}$ ) and CMX (strongly acidic, 1.8$3.8 \mathrm{Ocm}^{2} @ 25^{\circ} \mathrm{C}$ ) membranes were used (Ameridia, Somerset, NJ, manufactured by Astom Corp., Tokyo, Japan; data as supplied by the manufacturer). The membranes were configured as a 100-cell pair ED stack (Type 100, Deukum GmbH, Frickenhausen, Germany). In this stack configuration, the ED membranes are sandwiched between flexible frames with integrated turbulence promoters (polymeric netting) without additional gaskets. The cross-sectional active membrane area of the stack is about $99 \mathrm{~cm}^{2}$.

Electric power for desalting seawater samples in the ED system during the initial ED phase and the RO/ED phase was provided by a $7.5 \mathrm{~kW}$ DC power supply (PowerTen 4700 series). During the final ED phase, electric power was provided by a standard laboratory DC power supply. The power supplies were always operated in constant-current mode, with voltages that varied in response to the changing resistance of the ED stack. The limiting current density versus ED diluate conductivity was determined in the laboratory and confirmed on shipboard $^{1}$ for the ED stack and flowrates of diluate, concentrate, and electrode rinse solutions. Because ED continuously removes salts and lowers the conductivity of a sample, electric current must be manually decreased periodically to avoid heterolytic splitting of water that would result if the applied electric current reached or exceeded the limiting current at a given diluate conductivity $^{8}$. The manual stepwise potentiostatic operation chosen here avoids the issue that the overall electrical resistance of the stack may well vary from the conditions when the limiting current densities were determined, specifically in regard to the concentrate conductivity and the concentrate purge regimen which was not identical in the laboratory and on shipboard. The relation of stack electrical resistance and limiting current density is not unique. The voltage 
during a typical shipboard experiment with the 100-cell pair ED stack varied from approximately $250 \mathrm{~V}$ (power supply limit) initially to approximately 60-70 V at the end of the process.

During the July, 2006 cruise, continuous ED was used in the final ED phase for all but one sample, and pulsed ED was used for that sample by manually switching the DC power to the ED stack on/off at about two-second intervals. During the October, 2006 cruise, pulsed ED was used for all samples, using a KRDR Digi-Timer (input 12-120 Volt, ABB SSAC, Staten Island, NY) to automatically switch the DC power to the ED stack on/off at two-second intervals (with about $\pm 10 \%$ accuracy and $\pm 0.5 \%$ repeatability). These settings have not yet been optimized. The intervals were chosen based on the literature ${ }^{15}$ and on our experimental observations of the time needed to reach a steady voltage after a stepwise change in the ED current.

In the shipboard ED system, Iwaki MD40RLT seal-less centrifugal pumps were used to circulate the sample (diluate) and concentrate solutions through the ED system at a flow rate of $640 \mathrm{~L} \mathrm{hr}^{-1}$. An Iwaki MD20RLT seal-less centrifugal pump was used to circulate the electrode rinse solution through the ED system at a sufficient flow rate to avoid hydrostatic pressure gradients to the diluate and concentrate circuits. Flow rates on shipboard were determined volumetrically.

\section{Analytical methods}

On shipboard, conductivity and $\mathrm{pH}$ were measured at regular intervals during all three phases of the RO/ED process, using a hand-held, temperature-compensated conductivity meter (Oakton 300 Series) and an Orion 720A pH meter. Voltage and current readings were regularly taken directly from the DC power supply.

All samples for DOC analysis were collected in $40 \mathrm{~mL}$ glass sample vials. All samples were refrigerated until analyzed, and DOC analyses were completed within one week. DOC was 
measured using a Shimadzu TOC-VCSN high-temperature combustion analyzer (Shimadzu

Scientific Instruments, Columbia, MD) using standard protocols for seawater ${ }^{16}$. The system blank $^{17}$ of the Shimadzu analyzer was estimated to be $0.16 \mathrm{mg}$ TOC/L. This is the average difference between TOC measurements for purified laboratory water on the Shimadzu analyzer and a Sievers 800 carbon analyzer (GE Analytical Systems, Boulder, CO). The Sievers carbon analyzer uses a combination of photo- and chemical oxidation to break down DOM and has no inherent system blank.

\section{Results}

Overall results

An overview of nine experiments (from a total of 16) for recovery of DOM from seawater is given in Table 1. The selected experiments were all conducted on seawater samples that were collected in the Atlantic Ocean 170 - $350 \mathrm{~km}$ east-south-east of Savannah, Georgia. The focus of this report is the subset of experiments performed on the October, 2006 cruise (last four rows in Table 1). The results selected from the earlier July, 2006 cruise provide a basis for evaluating the use of pulsed electric current in the final ED phase. The first three samples (S7063, S706-5, and S706-6) were used in the previous publication ${ }^{1}$; however, some results from Table 1 of that paper are corrected here due to small $(<2 \%)$ changes in the volume of initial and final seawater samples compared to our earlier paper. As noted earlier in the paper, the initial volumes of all samples have been corrected for prior calibration errors and the conductivity of S706-3 has been corrected from 10.0 to $8.98 \mathrm{mS} \mathrm{cm}^{-1}$. The samples vary in location, depth, initial and final conductivities, and initial DOC concentrations. If the deepest sample (322 m) from the July cruise is neglected, then similar recoveries of DOC were obtained in July (61\% $77 \%)$ and October $2006(62 \%-72 \%)$. It is evident from these results that the use of pulsed 
electrical current in the final ED phase enables roughly 100-fold greater removal of salts while maintaining comparable recoveries of DOC.

The average mass ratio of residual sea salts to DOM in isolated samples of marine DOM can be estimated, if it is assumed that the mass concentrations of sea salts $\left(\mathrm{mg} \mathrm{l}^{-1}\right)$ and the conductivities of samples $\left(\mathrm{mS} \mathrm{cm} \mathrm{cm}^{-1}\right)$ are reduced by the same percentage during the RO/ED process and that $\mathrm{DOM} \sim \mathrm{DOC} \times 2$. For the first four samples in Table 1, all of which were processed using continuous electrodialysis in the final phase of the RO/ED process, the average ratio of residual sea salts to DOM is estimated to be around 180:1. In contrast, a ratio of 1.5:1 is estimated for the last four samples in Table 1, all of which were processed using pulsed electrodialysis in the final phase of the RO/ED process. When pulsed electrodialysis is used, the final isolated samples are sufficiently enriched in marine DOM that they can be studied using advanced chemical and spectroscopic methods, and the authors have already obtained some results of such measurements that will be published elsewhere. At present, the final isolated samples still contain more than $50 \%$ residual sea salts, which precludes accurate elemental analyses for $\mathrm{H}$ and $\mathrm{O}$. Further enhancements of the RO/ED method will be required before such analyses become routinely available.

\section{Detailed analysis of the removal of sea salts and water}

The removal of water versus the removal of sea salts (represented by a change in the product of conductivity and volume) during the RO/ED and pulsed RO/ED experiments for DOM recovery are shown in Figure 3. Data from all experiments appear in Figure 3 as two approximately linear segments, and there is no discernible difference between the two groups of experiments (with or without pulsed ED). The first linear segment, during which slightly less than $75 \%$ of sea salts are removed, is the initial ED phase of the coupled RO/ED process. 
Interestingly, even though the RO system is not pressurized during the initial ED phase, the volumes of samples decrease from $198 \mathrm{~L}$ to around $180 \mathrm{~L}$. Because ions are strongly solvated in aqueous solution, the transfer of cations and anions from the sample to the ED concentrate is accompanied by a corresponding transfer of $\mathrm{H}_{2} \mathrm{O}$ molecules. The second linear segment, during which nearly all remaining sea salts and water are removed, is the RO/ED phase of the coupled RO/ED process. The final ED phase, during which the effect of pulsed ED should be manifested, is not visible at the scale of Figure 3.

The results in Figure 3 can be better understood using a simple pedagogic example. Suppose a seawater sample is processed using the coupled RO/ED method, following the general protocol that is outlined in Figure 1. Suppose further that the volume and conductivity of the sample at the beginning of the process, at the end of the initial ED phase, the end of the RO/ED phase, and the end of the final ED phase are $\left(198 \mathrm{~L}, 50 \mathrm{mS} \mathrm{cm}^{-1}\right),\left(180 \mathrm{~L}, 15 \mathrm{mS} \mathrm{cm}^{-1}\right),(5 \mathrm{~L}$, $\left.15 \mathrm{mS} \mathrm{cm}^{-1}\right)$, and either $\left(5 \mathrm{~L}, 10 \mathrm{mS} \mathrm{cm}^{-1}\right)$ or $\left(5 \mathrm{~L}, 0.05 \mathrm{mS} \mathrm{cm}^{-1}\right)$, respectively. The percent removal of sea salts at the end of any stage in the process is given by the expression:

$$
100\left[1-\frac{(\text { Conductivity } \cdot \text { Volume })_{i^{\text {th }} \text { Stage }}}{(\text { Conductivity } \cdot \text { Volume })_{\text {initial }}}\right]
$$

At the end of the initial phase, $72.7 \%$ of sea salts have been removed. At the end of the RO/ED phase, $99.2 \%$ of sea salts have been removed. At the end of the final ED stage, either $99.5 \%$ (continuous ED) or $99.997 \%$ (pulsed ED) of sea salts have been removed. The improved desalting in the final ED phase (from $99.2 \%$ to either $99.5 \%$ or $99.997 \%$ ) cannot be seen at the resolution of Figure 3.

Figure 4 is presented to better visualize the impact of pulsed ED on the recovery of DOC (the principal objective of the study). Based on field measurements of volume and measured 
DOC concentrations of intermediate grab samples, the percent recovery of DOC can be tracked through the entire coupled RO/ED process. For the samples in Table 1, the average percent recovery of $\mathrm{DOC}$ at the end of the $\mathrm{RO} / \mathrm{ED}$ phase was $92 \% \pm 16 \%$, which is considerably greater than the final recoveries of DOC in Table 1. The variation of percent recovery of DOC with conductivity for intermediate points within the final ED phase is plotted in Figure 4. To focus on the comparison between experiments conducted with and without pulsed electric current in the final ED phase, the percent retention values for all experiments have been normalized to $100 \%$ at the beginning of the final ED phase, and only the results for the final ED phase are shown in Figure 4. The percent recovery of DOC slightly exceeded $100 \%$ for some of the intermediate data that are plotted in Figure 4; however, the discrepancies are within the precision of the combined DOC and volume measurements.

The data in Figure 4 fall into two distinct groups. For all samples that were processed using continuous (non-pulsed) ED in the final ED phase, the percent recovery of DOC began to decrease sharply at a conductivity of around $14 \mathrm{mS} \mathrm{cm}^{-1}$. For all samples that were processed using pulsed ED in the final ED phase, the percent recovery of DOC remained nearly constant until conductivity reached $1 \mathrm{mS} \mathrm{cm} \mathrm{cm}^{-1}$, after which the percent recovery of DOC dropped dramatically as conductivity was lowered further to $0.05-0.20 \mathrm{mS} \mathrm{cm}^{-1}$.

\section{Possible mechanism(s) of loss of DOC}

As shown previously ${ }^{9}$, continuous non-pulsed ED can be used to remove $\mathrm{SO}_{4}{ }^{2-}$ from water samples in which DOM has been pre-concentrated by RO. As ED was used to reduce the conductivity (including $\mathrm{SO}_{4}{ }^{2-}$ ), significant losses of DOM were observed. Those losses were at least partially prevented by maintaining conductivity at $>1 \mathrm{mS} \mathrm{cm}-1$ by periodic additions of $\mathrm{NaCl}$ to the sample. This observation at least suggests that the presence of a significant 
concentration of $\mathrm{Cl}^{-}$in the sample (sometimes termed a "pusher ion") as $\mathrm{SO}_{4}{ }^{2-}$ is being removed by ED somehow mitigates against losses of DOC. It was also previously $\operatorname{shown}^{1}$ for the first three samples in Table 1 that the greatest loss of DOC occurs during the final ED phase. Ratios of sea salts to DOM have already been estimated in this paper for the initial seawater samples (17,500:1), isolated DOM samples that were processed using continuous ED in the final ED phase (180:1), and isolated DOM samples that were processed using pulsed ED in the final ED phase $(1.5: 1)$. Because DOM is negatively charged at circumneutral $\mathrm{pH}$, the concentrated DOM is perhaps able to out-compete the more rapidly migrating, but now scarce, inorganic anions as conductors of charge through the boundary layers and the anion exchange membranes themselves. Loss of DOC by this mechanism is slowed by use of pulsed electrical current in the final ED phase. On the other hand, the DOC lost from the sample during final ED should appear in the electrodialysis concentrate, if the DOM penetrated the membrane. The DOC mass balance could not be closed in this way during final ED. The wt $\%$ of DOC recovered here is identical to the closure of the mass balance.

A closely related possibility is that DOM was deposited at the sample side of the ED anion exchange membranes during the final ED phase and was not completely recovered by rinsing with $0.01 \underline{\mathrm{M} \mathrm{NaOH}}$ (a step which was used only during the October, 2006 cruise). Such a loss mechanism suggests the possibility that DOM might be carried over from one sample to the next; however, mass balances of DOC do not support this scenario. Given the previously observed beneficial effect of $\mathrm{Cl}^{-}$in mitigating against losses of DOC, it is probable that any residual DOM from processing of one sample is flushed from the RO/ED system when the system is washed with $50 \mathrm{~L}$ of the next seawater sample (high $\mathrm{Cl}^{-}$concentration). Samples of the 50 L wash solutions were unfortunately not taken. 
The effects of $\mathrm{pH}$ and ionic strength on adsorption of natural organic matter (NOM) to an AMX anion exchange membrane were examined elsewhere ${ }^{18}$ and it was found that adsorption increases at high $\mathrm{pH}$ and low ionic strength. The authors concluded that, at higher ionic strength, $\mathrm{Cl}^{-}$ions in the background electrolyte out-compete NOM for anion exchange sites. They interpreted the greater adsorption of $\mathrm{NOM}$ at higher $\mathrm{pH}$ as evidence for high concentrations of phenolic hydroxyl groups in the NOM, even greater than the carboxyl content of the NOM. This explanation is inconsistent with most published data on the acidic functional groups of NOM and related materials ${ }^{19}$.

In any case, whether the lost DOC penetrated the membrane or deposited on the membrane, either process could be considered to be partially counteracted by periodical reequilibration with the bulk fluid on the sample (diluate) side of the membranes, especially in light of other work ${ }^{12}$.

Figure 5 shows a tentative concept that perhaps illustrates the function of pulsed ED in aqueous solutions with very low conductivity but relatively high DOC concentrations. Fluid dynamics (mixing vs. boundary layers), diffusion, and migration of ions in an electrical field are the main physical processes. It shall be assumed that a stagnant boundary layer exists near the membrane surface. It is appropriate to focus on the sample (concentrated DOC) side of the anion exchange membrane, because DOM is negatively charged under processing conditions used in this study. When electrical current is applied, charged species will experience a force according to their charge and the field gradient. Anions will migrate from the well-mixed bulk solution through the stagnant boundary layer towards the anion exchange membrane. Because small inorganic ions will migrate much more rapidly (at this point in the process, sulfate ions are the predominant remaining inorganic anion, data not shown) than large organic anions, it is likely 
that the membrane boundary layer is quickly depleted of these ions, thus forcing organic anions to play a larger net role in maintaining the electrical current through the membrane (thus disappearing from the sample) and/or inducing fouling of the membranes by high concentrations of organic ions. If the electric current is now turned off, then regeneration (re-mixing) of the boundary layer is expected (diffusional influx of inorganic ions and removal of organic ions to the bulk solution). Engaging the electrical current again, the inorganic ions will once again carry the electrical current. On/off pulses of a length sufficient to allow diffusional regeneration of the boundary layer may therefore be beneficial. It has been observed experimentally that establishing steady state in electrodialysis after a major change (for example a step increase of electrical current, or turning the current on/off) takes on the order of seconds or less. Therefore an interval of two seconds on/off was chosen as a reasonable time for regeneration of the stagnant boundary layer at the membrane as is also qualitatively suggested elsewhere 15.

\section{Other noteworthy observations}

During the October, 2006 cruise, the ED concentrate for the final ED phase was contained in a 24 L HDPE tank, rather than the original 230 L tank. This modification facilitated more frequent dilutions of the ED concentrate with RO permeate. Without frequent dilution, the accumulated salinity in the ED concentrate quickly exceeded that of the sample, and backdiffusion of salts down the concentration gradient became competitive with ED, greatly decreasing the efficiency of the ED process.

Somewhat different rinsing protocols were used in the two cruises. After every run during the July, 2006 and October, 2006 cruises, the RO system and all associated tubing and fittings were rinsed with $0.01 \underline{\mathrm{M}} \mathrm{NaOH}$. A gentle gravity-driven procedure was used on the July, 2006 cruise, and an average of $5.1 \mathrm{mg}$ of DOC was recovered (2.6\% of the average initial DOC 
in a sample). The ED stack was not rinsed in the July, 2006 cruise. In the October, 2006 cruise, a much more vigorous rinse was conducted by rapid circulation of the $\mathrm{NaOH}$ solution through the RO system; however, the average recovery of DOC only increased slightly to $6.6 \mathrm{mg}$. In the October, 2006 cruise, the ED stack was also rinsed vigorously using the same solution that had been used to rinse the RO system. On average, an additional $11.3 \mathrm{mg}$ of DOC was recovered from the ED system. Collectively, the average $17.9 \mathrm{mg}$ of DOC that was recovered by vigorous

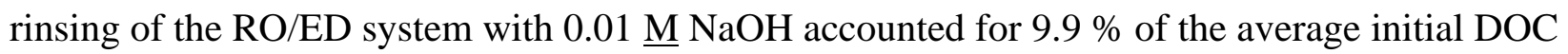
in a sample.

The temperature of all solutions generally rose during processing from ambient temperature $\left(26-27^{\circ} \mathrm{C}\right)$ to a maximum of about $35^{\circ} \mathrm{C}$ at the end of the RO/ED phase, and then declined somewhat to about $34^{\circ} \mathrm{C}$ at the end of the final pulsed $\mathrm{ED}$ phase. The temperature rise was in large part due to the significant input of electrical power for ED during removal of the bulk of the inorganic salts (initially as high as $4.5 \mathrm{~kW}$ ).

In the cruise of July, 2006, the sample was only circulated through the ED system during the initial ED phase. The transition from the initial ED phase to the RO/ED phase was marked by a sharp increase in salinity in the sample processing tank as completely unprocessed seawater from the void space in the RO system was mixed back into the partially desalted sample. This problem was eliminated in the October, 2006 cruise by circulating the sample through both the $\mathrm{RO}$ and ED systems during the initial ED phase.

During the RO/ED phase of operation, the rate of production of the permeate solution gradually increased from $1.3 \mathrm{~L} \mathrm{~min}^{-1}$ to $2.7 \mathrm{~L} \mathrm{~min}^{-1}$ as the conductivity (salinity) of the sample decreased. The conductivity of the RO permeate was generally in the range of $1 \%-2 \%$ of that 
of the sample (e.g., at the start of the RO/ED phase, conductivities of the sample and permeate solutions were around 15,000 and $300 \mu \mathrm{S} \mathrm{cm}^{-1}$ ).

\section{Conclusions}

$\mathrm{RO} / \mathrm{ED}$ processing has very significantly improved upon the state of the art in recovery of marine DOM from seawater for research in chemical oceanography and related issues such as global carbon balances. When the coupled RO/ED process uses continuous electric current in the final ED stage, the ratio of residual sea salts to DOM can be reduced from $17,500: 1$ to $180: 1$ with an average $76 \%$ recovery of DOC. This study has shown that the use of pulsed electric current in the final ED phase further lowers the ratio of residual sea salts to DOM to 1.5:1 - an improvement of more than two orders-of-magnitude - with no deleterious impact on the percent recovery of DOC. The much more efficient desalting of these samples (without further loss of DOM) enables direct post processing of concentrated marine DOM by freeze-drying, and facilitates the subsequent sophisticated analyses of marine DOM that are routinely applied to DOM from less saline source materials. Further improvements in the RO/ED process will be required to obtain truly low-ash samples that can yield accurate elemental analyses for all major components of DOM (C, H, O, N, S, and P).

\section{Acknowledgements}

This material is based upon work supported by the National Science Foundation (NSF) under Grants No. 0425624 and 0425603. Any opinions, findings, and conclusions or recommendations expressed in this material are those of the authors and do not necessarily reflect the views of NSF. 
The authors would like to express our sincere appreciation to Dr. Mary Rezac, who initiated the contact between scientists and engineers that made this work possible. The authors would like to thank Poulomi Sannigrahi for help at sea and in the laboratory and to especially thank Captain Raymond Sweatte and the excellent crew of the R/V Savannah for a great and productive cruise. 


\section{References}

${ }^{1}$ T.A. Vetter, E.M. Perdue, E. Ingall, J.-F. Koprivnjak, P.H. Pfromm, Combining reverse osmosis and electrodialysis for more complete recovery of dissolved organic matter from seawater, Separation and Purification Technology, 56(3) (2007) 383.

2 J. Hedges, J. Oades, Comparative organic geochemistries of soils and marine sediments, Organic Geochemistry, 27(7-8) (1997) 319.

${ }^{3}$ R. Benner, Chemical composition and reactivity, in Biogeochemistry of Marine Dissolved Organic Matter, D. A. Hansell and C. A. Carlson, eds., (2002) 59.

${ }^{4}$ E. M. Perdue, R. Benner, Marine organic matter, in Biophysico-chemical Processes Involving Natural Nonliving Organic Matter in Environmental Systems, N. Senesi, B. Xing, and P.M. Huang, eds., Wiley, (2008), in press.

${ }^{5}$ S. Sourirajan, Reverse Osmosis, Academic Press, New York, 1990.

${ }^{6}$ S.M. Serkiz, E.M. Perdue, Isolation of dissolved organic matter from the Suwannee River using reverse osmosis, Water Research, 24(7) (1990) 911.

${ }^{7}$ L. Sun, E.M. Perdue, J. McCarthy, Using reverse osmosis to obtain organic matter from surface and groundwaters, Water Research, 29(6) (1995) 1471.

${ }^{8}$ H. Strathmann, Ion-Exchange Membrane Separation Processes (Membrane Science and Technology Volume 9), Elsevier, Amsterdam, 2004.

${ }^{9}$ J.-F. Koprivnjak, E.M. Perdue, P.H. Pfromm, Coupling Reverse Osmosis with Electrodialysis to Isolate Natural Organic Matter from Fresh Waters, Water Research, 40(18) (2006) 3385.

${ }^{10}$ J.E. Drewes, J. Mitterwallner, S. Gruenheid, C. Bellona, A novel approach using reverse osmosis/electrodialysis (RO/ED) to concentrate and isolate organic carbon from water samples, Proceedings, American Water Works Association, Water Quality Technology Conference, Seattle, WA, November 2002, 252.

${ }^{11}$ V. Lindstrand, G. Sundström, A.S. Jönsson, Fouling of electrodialysis membranes by organic substances, Desalination, 128 (2000) 91.

12 B. Ruiz, P. Sistat, P. Huguet, G. Pourcelly, M. Araya-Farias, L. Bazinet, Application of relaxation periods during electrodialysis of a casein solution: Impact of anion-exchange membrane fouling, Journal of Membrane Science, 287 (2007) 41.

${ }^{13}$ Y.V. Karlin, V.N. Kropotov, Electrodialysis Separation of $\mathrm{Na}^{+}$and $\mathrm{Ca}^{2+}$ in a Pulsed Current Mode, Russian Journal of Electrochemistry, 31(5) (1995) 472.

${ }^{14}$ H.J. Lee, S.H. Moon, S.-P. Tsai, Effects of pulsed electric fields on membrane fouling in electrodialysis of $\mathrm{NaCl}$ solution containing humate, Separation and Purification Technology, 27 (2002) 89. 
${ }^{15}$ N. A. Mishchuk, L. K. Koopal, G. Gonzalez-Caballero, Intensification of electrodialysis by applying a non-stationary electrical field, Colloids and Surfaces A: Physicochemical and Engineering Aspects, 176 (2001) 195.

${ }^{16}$ K. Grasshoff, K. Kremling, M. Erhardt, Methods of seawater Analysis, Wiley-VCH, Weinheim, 1999.

${ }^{17}$ J.H. Sharp, R. Benner, L. Bennett, C.A. Carlson, R. Dow, S.E. Fitzwater, Reevaluation of High-Temperature Combustion and Chemical Oxidation Measurements of Dissolved OrganicCarbon in Seawater, Limnology and Oceanography, 38(8) (1993) 1774.

${ }^{18}$ D.H. Kim, S.-H. Moon, J. Cho, Investigation of the adsorption and transport of natural organic matter (NOM) in ion exchange membranes, Desalination, 151 (2002) 11.

${ }^{19}$ J.D. Ritchie, E.M. Perdue, Proton-binding study of standard and reference fulvic acids, humic acids, and natural organic matter, Geochimica et Cosmochimica Acta, 67(1) (2003) 85. 


\section{Tables}

Table 1: Overview of nine RO/ED experiments for the recovery of DOC from seawater on shipboard. Experiments performed during 10-06 are with pulsed ED.

\begin{tabular}{|c|c|c|c|c|c|c|c|c|c|c|}
\hline \multirow[t]{2}{*}{ Sample Description } & \multirow[t]{2}{*}{ के } & \multirow[t]{2}{*}{ कृ } & \multicolumn{2}{|c|}{$\begin{array}{c}\text { Seawater Volume } \\
{[\mathrm{L}]}\end{array}$} & \multicolumn{2}{|c|}{$\begin{array}{l}\text { Conductivity } \\
{\left[\mathrm{mS} \mathrm{cm}^{-1}\right]}\end{array}$} & \multicolumn{2}{|c|}{$\begin{array}{l}\mathrm{DOC} \\
{[\mathrm{mg} / \mathrm{L}]}\end{array}$} & \multirow{2}{*}{$\begin{array}{l}\text { DOC } \\
\text { [mg] }\end{array}$} & \multirow[t]{2}{*}{$\begin{array}{c}\text { Yield } \\
\text { of DOC } \\
{[\%]}\end{array}$} \\
\hline & & & initial & final & initial & final & initial & final & & \\
\hline $\begin{array}{l}\text { S706-3: Lat. 3105.9', } \\
\text { Long. } 77^{\circ}-27.2^{\prime} \\
20 \text { m depth, 7-20-2006 }\end{array}$ & $\diamond$ & No & 198 & 6.84 & 44.2 & 8.98 & 1.01 & 21.31 & 200 & 77 \\
\hline 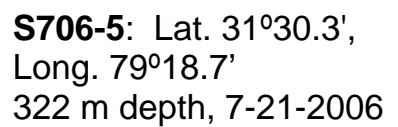 & $\square$ & No & 198 & 8.14 & 51.2 & 8.75 & 0.63 & 14.50 & 125 & 95 \\
\hline $\begin{array}{l}\text { S706-6: Lat. 3129.5', } \\
\text { Long. 79⒚2' } \\
77 \text { m depth, 7-22-2006 }\end{array}$ & $\Delta$ & No & 198 & 5.90 & 53.2 & 10.79 & 0.94 & 20.49 & 187 & 68 \\
\hline $\begin{array}{l}\text { S706-7: Lat. 3130.1', } \\
\text { Long. 79⒚4' } \\
20 \text { m depth, 7-24-2006 }\end{array}$ & $x$ & No & 198 & 7.00 & 52.6 & 9.45 & 1.03 & 17.03 & 204 & 61 \\
\hline $\begin{array}{l}\text { S706-8: Lat. } 31^{\circ} 30.0^{\prime} \\
\text { Long. } 79020.3^{\prime} \\
20 \text { m depth, 7-25-2006 }\end{array}$ & 0 & Yes & 198 & 8.48 & 51.2 & 0.187 & 0.95 & 14.43 & 188 & 68 \\
\hline $\begin{array}{l}\text { S1006-2: Lat. } 31^{\circ} 32.8^{\prime}, \\
\text { Long. } 79^{\circ} 14.3^{\prime} \\
20 \text { m depth, 10-25-2006 }\end{array}$ & $\bullet$ & Yes & 198 & 5.89 & 51.3 & 0.050 & 0.95 & 18.36 & 189 & 70 \\
\hline $\begin{array}{l}\text { S1006-3: Lat. } 31^{\circ} 32.6^{\prime}, \\
\text { Long. } 799^{\prime} 15.7^{\prime} \\
20 \text { m depth, 10-26-2006 }\end{array}$ & i & Yes & 198 & 4.78 & 54.3 & 0.096 & 0.90 & 20.86 & 178 & 72 \\
\hline $\begin{array}{l}\text { S1006-4: Lat. } 31^{\circ} 32.5^{\prime}, \\
\text { Long. } 79^{\circ} 13.8^{\prime} \\
84 \text { m depth, } 10-26-2006\end{array}$ & $\boldsymbol{\Delta}$ & Yes & 198 & 7.27 & 56.2 & 0.099 & 0.79 & 13.11 & 157 & 72 \\
\hline $\begin{array}{l}\text { S1006-5: Lat. } 31^{\circ} 32.5^{\prime} \text {, } \\
\text { Long. } 79^{\circ} 13.8^{\prime} \\
84 \text { m depth, } 10-27-2006\end{array}$ & $\diamond$ & Yes & 198 & 4.97 & 56.4 & 0.075 & 0.81 & 15.81 & 160 & 62 \\
\hline
\end{tabular}




\section{Figures}




\section{Preliminary Handling}

- Retrieve a seawater sample and filter the sample using a $0.45 \mu \mathrm{m}$ filter discard the first $20 \mathrm{~L}$ of filtered sample.

- Flush the RO/ED system with $50 \mathrm{~L}$ of filtered sample.

- Fill the sample processing tank with $200 \mathrm{~L}$ of filtered sample.

\section{Initial ED Phase}

- While circulating the sample through both the RO and ED systems, engage the ED system to decrease the conductivity of the sample to around $15 \mathrm{mS} \mathrm{cm}^{-1}$.

\section{RO/ED Phase}

- While circulating the sample through both the RO and ED systems, engage the RO system at a pressure of $1725 \mathrm{kPa}$ to remove water until the targeted final volume is reached. Regulate the ED current to control the rate of desalting and maintain a conductivity of around $15 \mathrm{mS} \mathrm{cm}$.

- Turn off the RO system and drain it completely, transferring all recovered sample to the sample processing tank.

\section{Final ED Phase}

- Use pulsed ED, cycled on/off at $2 \mathrm{~s}$ intervals, to reduce conductivity to $\sim 50 \mu \mathrm{S} \mathrm{cm}^{-1}$.

\section{Collect and Store DOM Samples}

- Drain all sample from the sample processing tank, ED system, and all connecting tubing. Freeze immediately.

- Circulate $0.01 \underline{\mathrm{M}} \mathrm{NaOH}$ through the RO system to recover adsorbed DOM, and drain completely.

- Circulate the same $0.01 \underline{\mathrm{M}} \mathrm{NaOH}$ solution through the ED system to recover adsorbed DOM, drain completely, and freeze immediately.

Figure 1: General protocol for shipboard isolation of marine DOM using the RO/ED method. 


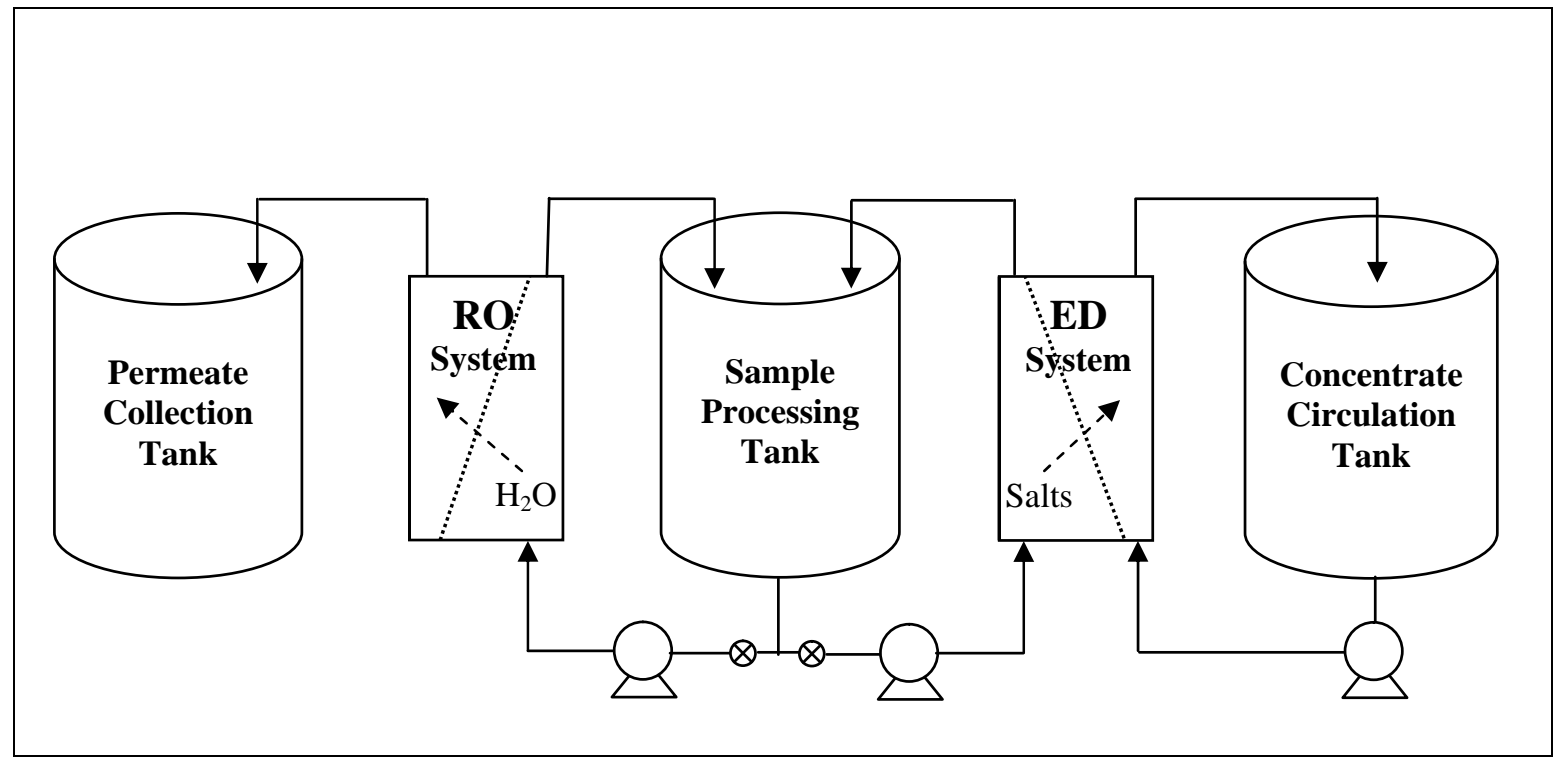

Figure 2: System for recovery of DOC from seawater using reverse osmosis and pulsed electrical current electrodialysis. 


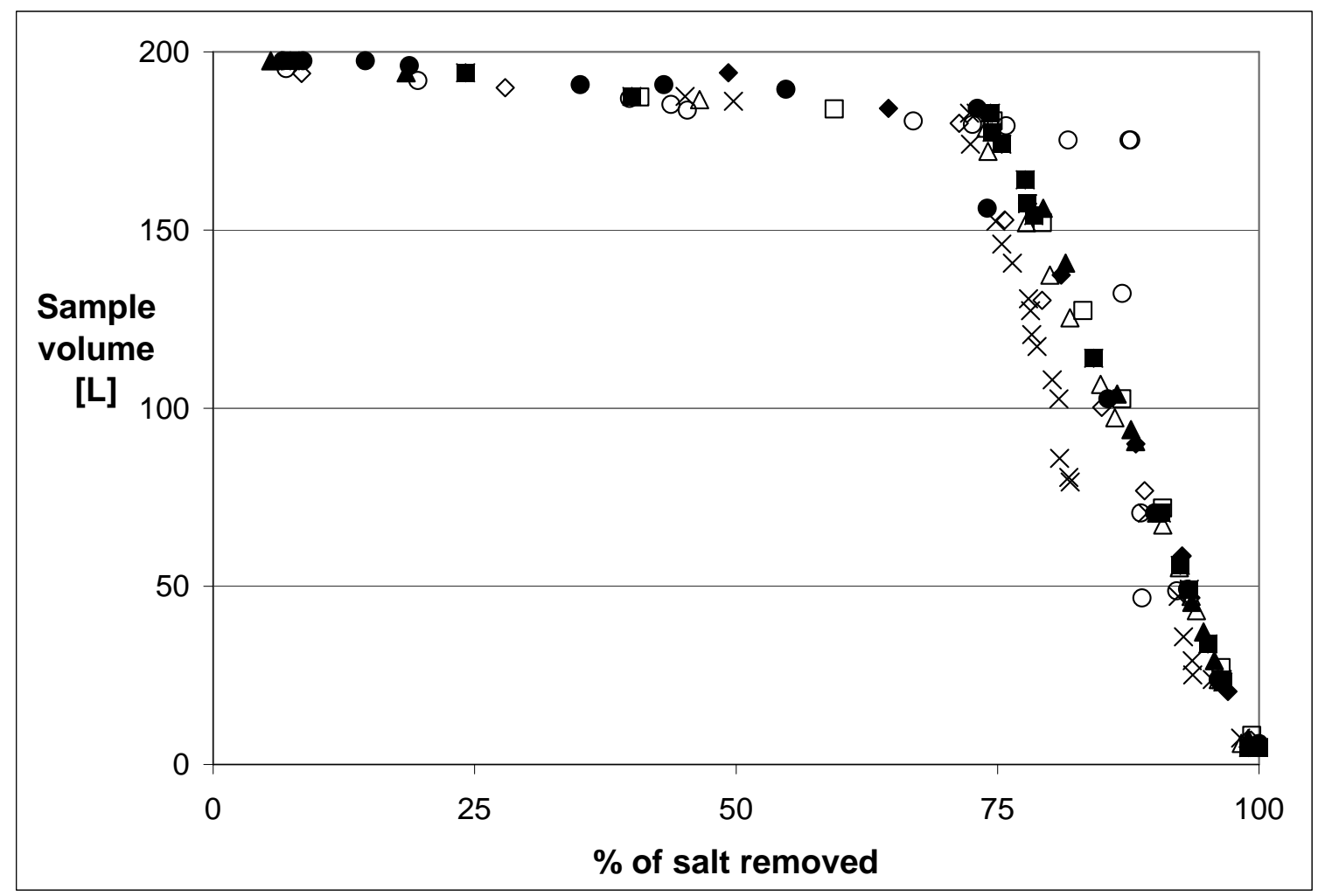

Figure 3: Reduction of the sample volume and sea salts (tracked by the product of conductivity and volume) during RO/ED processing of seawater samples. Symbols represent individual samples, as noted in Table 1. Solid symbols and open circles represent experiments performed with pulsed electrical current ED. Open symbols (excluding open circles) represent experiments performed with non-pulsed ED. 


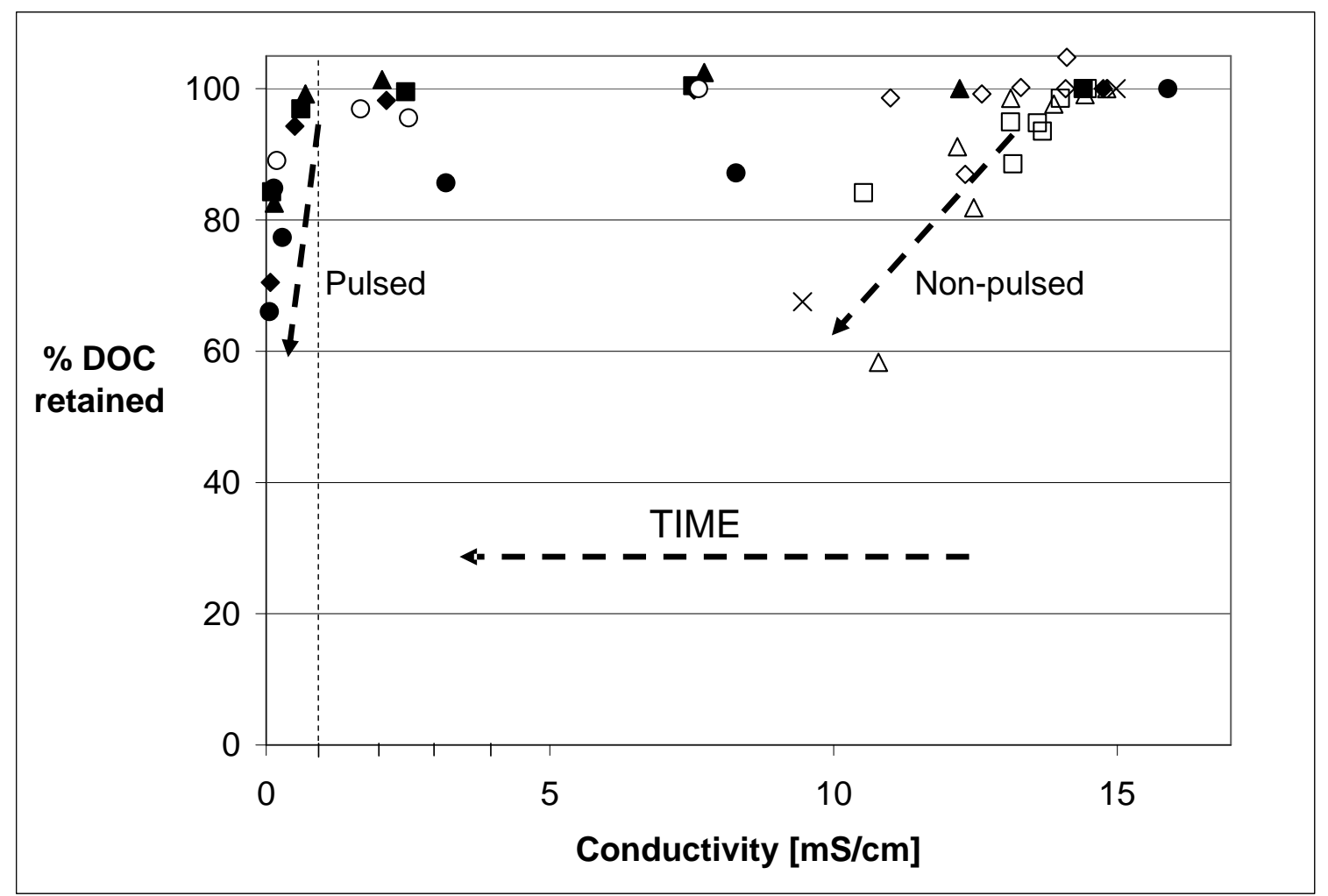

Figure 4: Retention of DOC during nine experiments on shipboard. Symbols represent individual samples, as noted in Table 1. Solid symbols and open circles represent experiments performed with pulsed electrical current ED. Open symbols (excluding open circles) represent experiments performed with non-pulsed ED. 


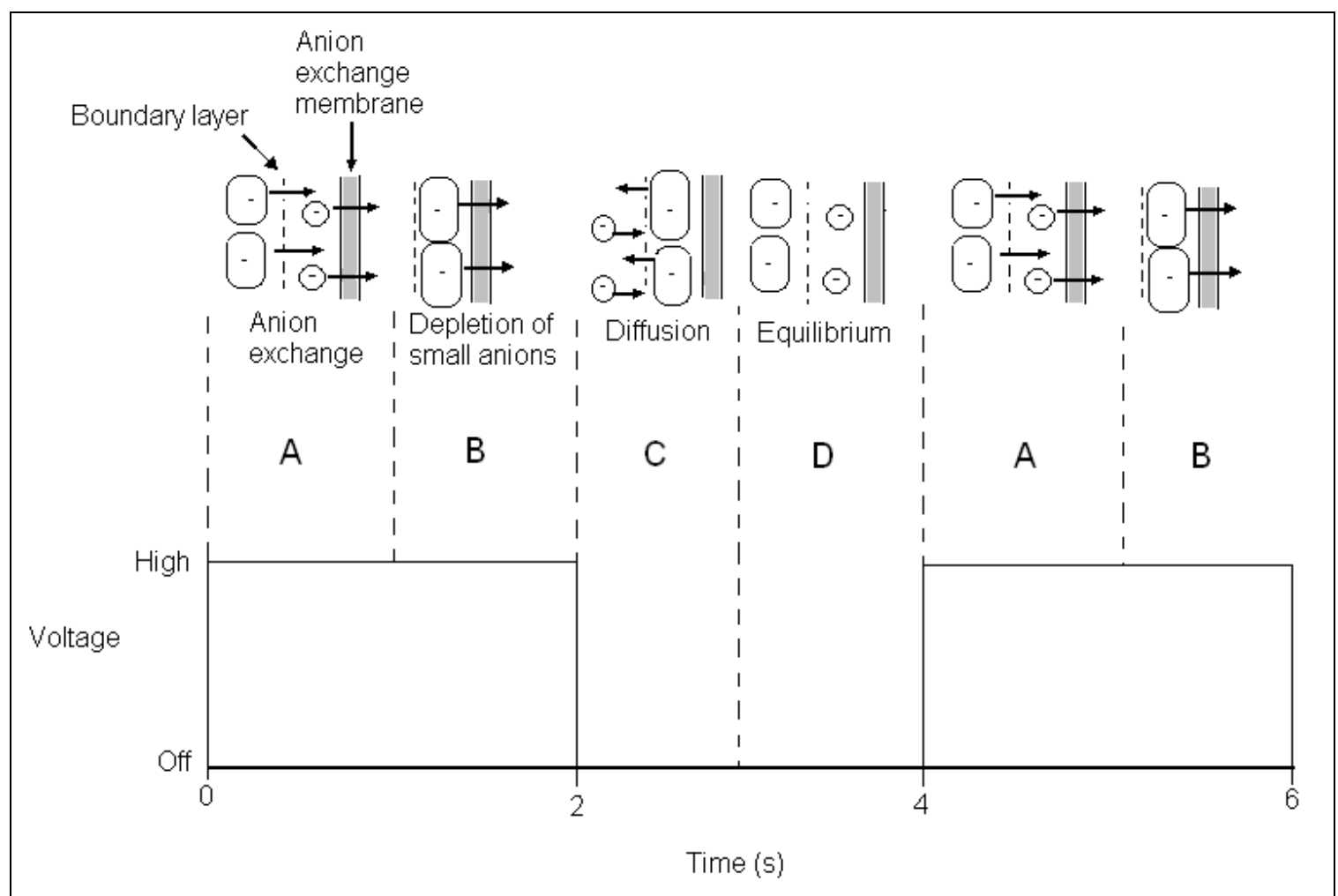

Figure 5: Expected occurrence of ions in the boundary layer of anion membrane during pulsed (on/off) electrical current. The letters (A, B, C, D) represent the different expected phases that the ions go through during the pulsed electrical current. A pulse of two seconds was used in these experiments. The smaller molecules represent the inorganic anions, while the larger molecules represent the larger DOC molecules. 